\title{
I got burnt once (IGBO)
}

\author{
"Do not dwell in the past, do not dream of the future, \\ Concentrate the mind on the present moment." \\ Dalai Lama
}

Decision-making in general practice is a complicated affair and seems to be one of those skills that get more difficult as one gains more experience and knowledge. After some years in practice, several adverse advents have almost always happened which may "colour" one's judgement.

As time goes by, a general practitioner starts to take many more peripheral factors into consideration when making diagnoses and decisions. Some of the strongest influences on our decision-making may be previous adverse events and experiences. These are the mistakes we made in treatments and the operations that went wrong.

We are also strongly influenced in the way we practice when a complaint has been made against us. The doctor naturally becomes extra cautious when a similar illness or situation arises again. The patient either gets over-investigated or referred unnecessarily to avoid an intervention that might lead to the same complication, otherwise known as practising Reassurance Medicine.

As litigation increases, we also move more and more into this avoidance behaviour called Negative Defensive Medicine. After reading about the cases of malpractice against doctors, I believe we all have sleepless nights on the basis of "therebut-for-the-grace-of-God-go-I." I find myself becoming extra careful in both my prescribing habits and the procedures I perform.

In a recent case, a breast lump was missed by a general practitioner. The reason was that he had previously been unjustly accused of inappropriately touching a patient during an examination. Following this incident he had, understandably, become cautious with intimate examinations. This resulted in him missing a breast lump as he had not re-examined a patient, who had already been screened by a nurse.

Long ago, one of my partners asked me to show him how to operate on a Meibomian cyst in the upper eyelid of one of his patients. I set up the eye tray with the Meibomian clamp and fine curette and put anaesthetic eye drops in the patient's eye. I then injected a small amount of local around the cyst. After this, I asked the patient to open her eye and to my horror the eye was completely white. I had injected the local through the eyelid into the eyeball and underneath the cornea. She had not felt it because I had put the local drops into the eye at the beginning. I was appalled at the sight of this completely white opaque eye staring at me. I had blinded her.

With a shaking hand, I phoned my favourite ophthalmic surgeon who reassured me that no harm would come from the local anaesthetic. He asked me to send her over to his office. She recovered in a day and had no adverse effects.

One of the most common adverse reactions in general practice is an allergic reaction to an injection. A few years ago, I gave a patient an injection of Diclofenac in my room. He then promptly dropped like a stone, unconscious, to the floor. It was a syncopal attack and after a few minutes he recovered. He actually recovered much quicker than his shaken doctor whose pulse rate remained fairly high for the rest of the morning, although the tightness and the choking feeling in my chest subsided after about an hour. I then found myself somewhat reluctant to give injections for the following few weeks.

Another case I was involved in also occurred a few years ago when a patient in our clinic was sent to our procedure room for a stress ECG for a routine insurance medical. He was a fit thirty-nine-year-old marathon runner and after a minute or two of running he had a heart attack on the treadmill. Although we have full resuscitative equipment with a crash cart and defibrillator as well as trained nurses in our rooms, we all found ourselves rather reluctant to do stress tests for a while.

Adverse events and reactions have an extraordinary effect on our subsequent decision-making in similar cases or situations. These reactions may be out of proportion to the actual condition, but psychologically they may be difficult to overcome. This happens in all disciplines of medicine and over time our confidence usually returns, but for some mishaps the doctor may completely lose his nerve.

I never operated on a Meibomian cyst again.

Chris Ellis is a family physician in Pietermaritzburg, KwaZulu-Natal

Email: cristobalellis@gmail.com 